\title{
Cardiovascular Risks Association with Circadian Rhythm Disruption in Shift Workers
}

\author{
Prerna Goyal1, \\ ${ }^{1}$ Department of Medicine, RG stone and Super Speciality Hospital, \\ Ludhiana, Punjab, India628687
}

Ind J Car Dis Wom 2021;6:86-87.

Shift work, defined as any work that is performed apart from a regular and traditional daytime work schedule, has been a matter of concern since a long time due to its negative impact on health and quality of life. ${ }^{1}$ Literature review by Rosa et al has shown that shift work involves an alteration in psychophysical homeostasis, with a decrease in performance. ${ }^{2}$ It is an obstacle for social and family relationships, as well as a risk factor for stress, sleep disorders, metabolic disorders, diabetes, cardiovascular disorders and breast cancer. A meta-analysis of 21 studies involving 173,010 participants found a nonlinear relation between shift work and cardiovascular disease (CVD) risk, which seemed to appear only after the first 5 years of exposure. Shift workers had 17\% higher risk of any CVD event among than day workers. ${ }^{3}$

The round-the-clock demands of health care services, coupled with the shortage of health care providers in many parts of the world, have made shift work widespread among health care workers, especially the nursing staff. ${ }^{4}$ Nursing work is a kind of job that requires high concentration, and the shift work system is an unavoidable attribute of nursing job. This makes nurses very vulnerable to circadian rhythm sleep disorders (CRSD), which are defined as the alteration in the 24-hour biological clock of humans, resulting in physical, clinical, and psychiatric symptoms. ${ }^{5}$

The American Academy of Sleep Medicine has published practice parameters for the clinical evaluation and treatment of CRSD. ${ }^{6}$ Shift work causes desynchronization of circadian rhythms, which can lead to various health-related problems such as neoplasia, cardiovascular diseases (hypertension, left ventricular hypertrophy, coronary heart disease and myocardial infarction), and digestive troubles, because of the alteration in the gut microbiota, fatigue, cancer, neurological disorders (depression, anxiety, Alzheimer's, etc.)., ${ }^{7.8}$

Data from earlier studies reported the impact of circadian rhythm disruption on cardiovascular health. Circadian rhythms are involved in controlling inflammatory processes

DOI https://doi.org/ 10.1055/s-0041-1732506
Address for correspondence Prerna Goyal, MD, Department of Medicine, RG stone and Super Speciality Hospital, Ludhiana 141002, Punjab, India (e-mail: Drprerna156@gmail.com).

and metabolisms, which can influence the pathology of arteriosclerosis and thrombosis. Targeting the circadian machinery in the vascular system with novel therapeutic molecules for the prevention and treatment of cardiovascular diseases has also been published. ${ }^{9}$

Interference in the normal blood pressure (BP) values of the nurses working during night shifts have been observed. Circadian rhythm variations have been found to be reduced during ambulatory blood pressure monitoring (ABPM) in night staff nurses. The exact mechanism of shift work and its impact on cardiovascular health is not yet clear but a little is known, in that nighttime shift working is considered hazardous to health, possibly due to its direct impact on our body's biological and physiological system, thereby contributing to the risk of CVD. This can possibly be due to a drop in melatonin synthesis during shift work. The latter is able to lower BP and reduce coagulability by itself. Moreover, lack of rest and reduction in vigilance combined with sleep deprivation can lead to stress, thus becoming a direct risk factor in CVD. ${ }^{2}$ Associations between low high-density lipoprotein (HDL) levels and recent night shifts, and increase in CRP levels and length of rotating night shifts, have also been reported. Although the data on the relationship between shift work and lipid parameters have been variable, it has been consistently reported that shift workers do show unfavorable alterations in lipids levels, a prominent risk factor of CVD. ${ }^{10}$ The effects of night shift work on sex hormones have also been shown in the underlying pathophysiology of shift work's association with cancers and CVD. Epidemiological evidence coupled with findings from in vitro and in vivo studies support the role of this pathway. ${ }^{4}$

In the study conducted at ICJM, Lucknow, published in this issue of the journal, authors recruited 25 night shift work nurses (NSWN) and 25 day shift work nurses (DSWN), and measured their seven days/24 hour ABM. ${ }^{11}$ Mean BP in NSWN was found to be higher. Misaligned body clock was observed

(c) 2021. Women in Cardiology and Related Sciences.

This is an open access article published by Thieme under the terms of the Creative Commons Attribution-NonDerivative-NonCommercial-License, permitting copying and reproduction so long as the original work is given appropriate credit. Contents may not be used for commercial purposes, or adapted, remixed, transformed or built upon. (https://creativecommons.org/licenses/by-nc-nd/4.0/).

Thieme Medical and Scientific Publishers Pvt. Ltd. A-12, 2nd Floor, Sector 2, Noida-201301 UP, India 
in $52 \%$ NSWN and only $8 \%$ in DSWN, as measured by circadian rhythm test; therefore, severity of circadian rhythm disruption was found to be more in NSWN.

Few previous studies on this subject have reported higher nocturnal BP readings and less nocturnal BP fall among night shift workers, but they used a fixed time period to describe sleep and awake periods without corrections for the real period of awake and sleep. They also found higher mean BP readings during the 24 -hour shift work, including values considered as hypertensive in few or some periods. The current study performed a comprehensive analysis of cardiovascular alterations, mainly BP levels, in young nurses in critical care unit by ABPM and circadian rhythm questionnaire. The authors observed that nurses working in night shifts showed reduced circadian rhythm variations in $\mathrm{BP}$, and have higher mean 24-hour systolic, diastolic, and sleeping time diastolic BP levels compared with those working during the daytime. This might be due to lack of sleep, which further elevated mental stress. Therefore, authors concluded that working in the critical care unit during the night hours leads to abnormal blood pressure in night shift working nurses, suggesting that this type of work, in which sleep is disturbed and leads to mental stress, could be a direct risk factor in CVD.

In a study from Bangalore, including 130 nurses, the prevalence of shift work disorder was found to be $43.07 \%$. Headache, back pain, gastritis, and menstrual disorders were the most common complaints, which are found in 78 (60.0\%), 75 (57.6\%), 42 (32.3\%), and 39 (30.0\%) cases, respectively. Anxiety and depression was found in 23 (17.6\%) and 31 (23.8\%) individuals, respectively. A significant association of shift work disorder with increasing age, more number of nights worked in a year, and longer duration of working hours were noted. ${ }^{7}$ Another study which included data of 116,429 female registered nurses from the Nurses' Health Study II (NHSII) elucidates the relationship between the circadian program, its molecular mediators, and CVD risk. ${ }^{10}$

It can be concluded that shift work, in which circadian rhythm is disturbed, leads to abnormal blood pressure in night shift working staff. This could lead to mental stress, which is a direct risk factor for CVD. A high prevalence of shift work disorder calls for urgent efforts to reduce the adverse health effects associated with shift work. Several ways by which CRSD due to working hours can be overcome include the following: behavioral changes, clockwise rotating shifts, taking a nap before work, modifying the environment by avoiding light if going to sleep, but approaching light if you have to wake up, resetting the sleep schedule, and taking melatonin. ${ }^{6}$

\section{Conflict of Interest}

None declared.

\section{References}

1 James Mclntosh. The impact of shift work on health. Medical News Today. Available at: https://www.medicalnewstoday. com/articles/288310. Accessed June 12, 2020

2 Rosa D, Terzoni S, Dellafiore F, Destrebecq A. Systematic review of shift work and nurses' health. Occup Med (Lond 2019;69(4):237-243

3 Torquati L, Mielke GI, Brown WJ, Kolbe-Alexander T. Shift work and the risk of cardiovascular disease. A systematic review and meta-analysis including dose-response relationship. Scand J Work Environ Health 2018;44(3):229-238

4 Yau A, Haque M. Shiftwork association with cardiovascular diseases and cancers among healthcare workers: a literature review. Medeni Med J 2019;34(4):387-395

5 Jadmiko AW, Firdausi R. Circadian rhythm sleep disorder among nurses in emergency department: a systematic review. Indonesian J Med 2018;3(1):27-32

6 Morgenthaler TI, Lee-Chiong T, Alessi C, et al. Standards of PracticeCommittee of the American Academy ofSleepMedicine. Practice parameters for the clinical evaluation and treatment of circadian rhythm sleep disorders. An American Academy of Sleep Medicine report. Sleep 2007;30(11):1445-1459

7 Anbazhagan S, Ramesh N, Nisha C, Joseph B. Shift work disorder and related health problems among nurses working in a tertiary care hospital, Bangalore, South India. Indian J Occup Environ Med 2016;20(1):35-38

8 Haus E, Smolensky M. Biological clocks and shift work: circadian dysregulation and potential long-term effects. Cancer Causes Control 2006;17(4):489-500

9 Man AWC, Li H, Xia N. Circadian rhythm: potential therapeutic target for atherosclerosis and thrombosis. Int $\mathrm{J}$ Mol Sci 2021;22(2):676

10 Johnson CY, Tanz LJ, Lawson CC, Schernhammer ES, Vetter C, Rich-Edwards JW. Night shift work and cardiovascular disease biomarkers in female nurses. Am J Ind Med 2020;63(3):240-248

11 Fatima G, Jha A, Khan MA. Disruption in circadian rhythm increases cardiovascular disease risk factors in shift working nurses. Ind J Car Dis Wom 2021. Doi: 10.1055/s-0041-1732508 